\title{
Study of the Performance of Multi-hop Routing Protocols in Wireless Sensor Networks
}

\author{
Nouredine Seddiki \\ Dep of Science, University of Bechar \\ Bechar, Algeria
}

\begin{abstract}
Currently in the literature, there are quite a number of multi-hop routing algorithms, some of which are subject to normalization.

Routing protocols based on clustering provide an efficient method for extending the lifetime of a wireless sensor network. Except that much of the research focuses less on communication between the Cluster-Head ( $\mathrm{CH})$, the nodes and the base station, and gives even less importance to the influence of the type of communication on the Life of the network.

The aim of this article is to make a comparative study between some routing algorithms. Since they are not based on analytical models, the exact evaluation of some aspects of these protocols is very difficult. This is the reason why we make simulations.

To study their performance. Our simulation is done under NS2 (Network Simulator 2). It allowed to obtain a classification of the different routing algorithms studied according to one of the metrics such as the loss of message, and the lifetime of a network.
\end{abstract}

Keywords-network sensors; routing protocol; simulation; NS2; d network lifetime

\section{INTRODUCTION}

For many and various reasons,nowadays and in our daily lives, the evolution of technology ensures that any change in appearance in any environment,is detected, measured and collected through small electronic components called nodes sensors. The sensor nodes have the distinction of being inexpensive compared to traditional sensors and possess limited energy resources for processing, computation and transmission.

In order to study a given phenome No in a given environment, it is necessary to install a wireless sensor network, scattering the ma number of nodes sensors tos can the entire space in question.

In wireless sensor networks, the energy used for the communication of the data captured by nodes,is very high, compared to that used for any other operation. That is why we felt it necessary to give a look to this component.

The minimization of the energy consumption and the extension of the life time of the network, still one of the biggest concerns of researchers in this field. Whya hierarchicaltopology?Whyclustering? And why is a multihopcommunication?These are three questions to be answered,in order to underst and completely the choice of protocols sample we collected.

In a flat topology,each node maybe both a sensor, sink and gate way, all nodes communicate with each other, so that

\author{
Bassou Abedsalem \\ Dep of Science, University of Bechar \\ Bechar, Algeria
}

communications traffic is very dense and there fore,there is a high consumption of energy, which reduces the lifetime of the network, even up to its exhaustion

To this end, the routing protocols based on groupings of clustered nodes, have been, introduced to provide an effective method to extend the lifetime of a wireless sensor network, and by reducing the communication traffic, which was much denser in a flat topology. First, let us focus only on the network topology. Figures Fig. Ia and Ib Fig illustrate a remarkable reduction in communication traffic between the two topologies, flat and hierarchical[8].

Hence, the choice of the hierarchical topology, where the role of a cluster member nodes can be summarized in the detection information from their environment and their communication to the cluster-head.The cluster-head is for its part aggregates this data and sends it to the base station.

Following the same strategy to achieve the same goal of energy consumption reduction for the extension of the life time of the network, attention, was given to the type of communication used during data exchange between the sensor nodes, ClusterHeads and the base station, for this their has been a shift from communications ata Single Hop to multi-hop communications. Figures, Fig.I.c, Fig.I.d.and Fig.I.e,present the various possible multi-hop communications in a hie rarchical topology.

Hence, the choice of multi-hop communications.

This document provides a comparative study of some multi-hop routing protocols in wireless sensor networks. It is, organized into four parts as follows:

The first part is a general introduction, in which the characteristics of wireless sensor networks (WSN), are mentioned. The second part is a state of the art: a literature review. As for the third part, it includes a comparison between some protocols and our synthesis drawn from this comparison. To close this document, part fourth, representing a conclusion that justifies that, the chosen protocol, is the most performance in the lot of protocols that we have chosen in our comparative study.

\section{RELATED WORKS}

For the reasons explained above, in this section we will outline some multi-hop routing protocols.

\section{A. KOCA (K-hop Overlapping Clustering Algorithm)}

Mustafa A.YOUSSEF Adel Youssefand MohammedF.YOUNIS [2] propose in 2009 an original algorithm 

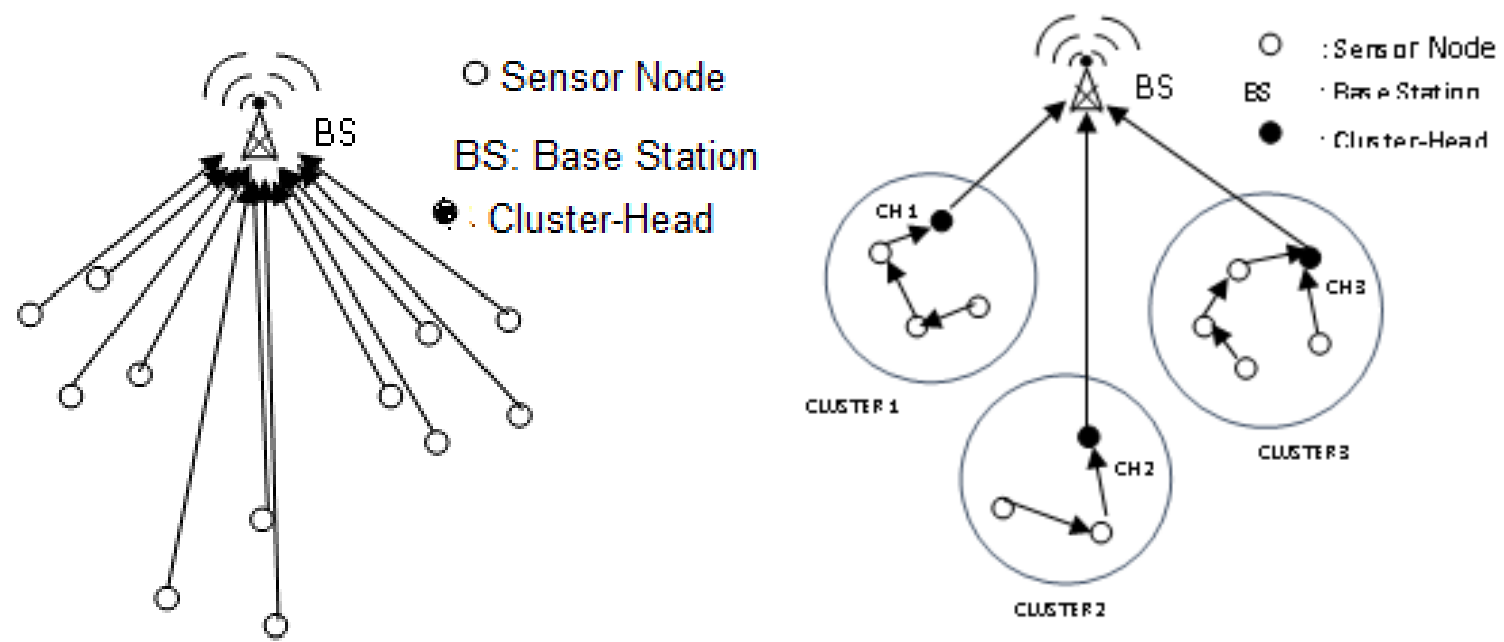

Fig.1.a : Single Hap withaut Cus tering

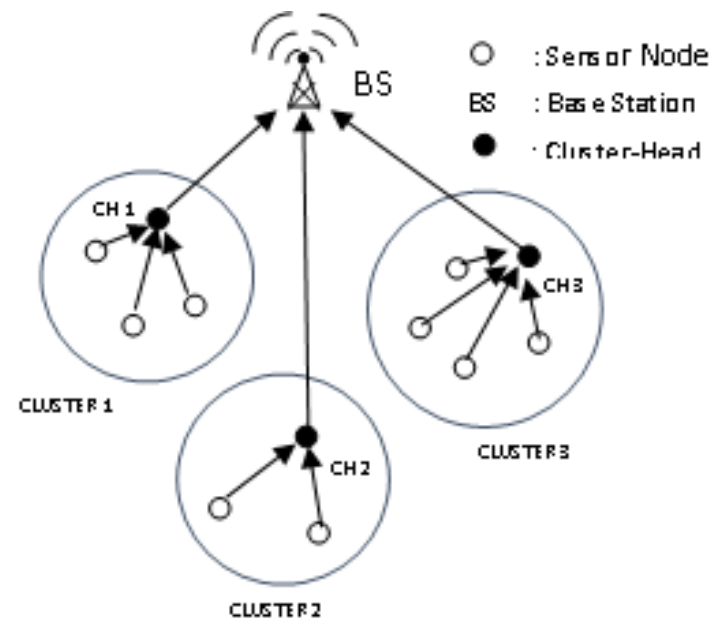

Fig.1.c: Imtra Clus ter Multi Hap Cammuniatian

Fig.1b : Single Hap with Clust ering

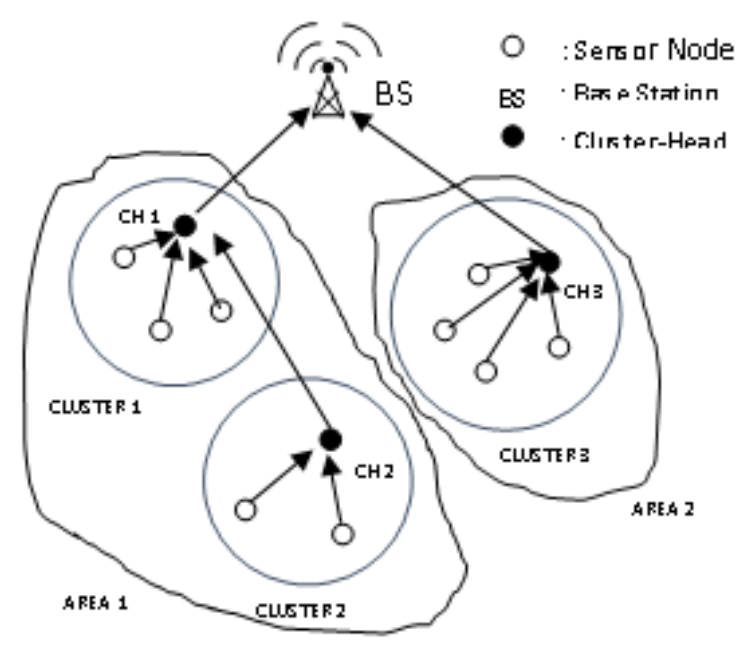

Fig. 1.d : Inter. Cluster Multi Hap Cammunicgtian

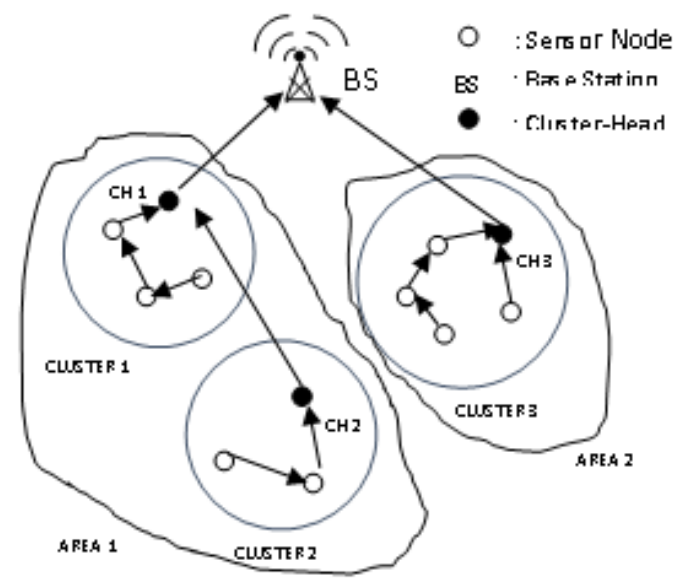

Fig. 1.e : Bath Intra. and Inter. Qus ter Multi Hap Cammunictian

Fig. 1: (a) Single Hop without Clustering (c) Intra Cluster Multi Hop Communication (b) Single Hop with Clustering (d) Inter. Cluster Multi Hop Communication (e) Both Intra. and Inter. Cluster Multi Hop Communication 
to build Over lapping clusters (overlapping) to reinforce the robustness of the network and to respond to specific issues, namely the inter-cluster transmission,node localization and time synchronization.

The overlapping clusters construction in a distributed manner is an NP-hard problem; the protocol KOCA solves this problem in arandom and distributed way. KOCA,be declared regardless of complexity of the network size.

The overlapping clusters training problem is formulated by KOCA by building a set of cluster-Heads satisfies the three conditions of coverage,overlapping and connectivity

\section{B. LMEEC (Layered Multi-Hop Energy Efficient Cluster- based)}

Manel Khelifi and Assia Djabelkhir [3] proposed in 2012, MEC, a new multi-hop clustering protocol based on layered and energy efficiency, which offers a new way to reduce the energy consumption of sensors.

In order to provide flexibility for routing data through the network, LMEEC introduced a layered topology for the network nodes according to the number of hops that each of them takes to reach the base station. Thus, to achieve high energy efficiency and increase network scalability, sensor nodes are organized into clusters. To this end, they define a new, grouping mechanism node into clusters. This mechanism ensures distribution of the workload of sensor nodes by structuring them into unequal size clusters. Then the cluster-Heads communicate the data collected from the network to the base station. The clusters Head are periodically selected according to weight. This weight is calculated so that the number of Cluster-head increases approaching the base station[9].

Therefore, the further cluster of the base station have the smaller sizes.

The execution of LMEEC is periodically established in threephases.The first is the network configuration,while the second ensures the election of cluster-heads and the formation ofclusters.Data communication is the third phase of the protocol.

\section{MCR (Multi-hop Clustering Routing Protocol)}

S. Koteswara Rao, M. and T. SailajaMadhu [1], proposein 2012a protocol Clustering, called Multi-hop Clustering Routing Protocol(MCR), based on the use of Gate way nodes to achieve delivery data to are motebase station with are as onablecost in energy.

The MCR protocol uses a principle of inter-cluster transmission with two jumps,ie, $\mathrm{CH}$ snodesdo not communicate directly with the well,butthey usean intermediate node(Gateway) that is located in an area covered by the base station.For nodes not covered by the well,the protocol proposes to use the same principle as the HEED protocol for building clusters.

\section{D. $k h L C H$}

The contribution of Khaled BOUCHAKOUR [4] in 2012 consists of a hierarchical routing protocol, called $\mathrm{KhLCH}$ (K.hop Layered Clutering Hierarchy), which aims minimizing energy consumption, scalability and reduced data delivery time. His solution uses K.Clusters formed on a restructured layered network; it allows for multi-hop communications, intra. and inter. Clusters, and collaborative data aggregated at Cluster-head and Gateway Nodes.

His solution is initially minimizing energy consumption and scalability; nodes are organized into layers according to their minimum distances, number of hops, the base station (the basic idea of LCH protocol). Then, these nodes are, organized into $\mathrm{k}$ clusters where each member node is either Clusterheadbe a member to k-hop $\mathrm{CH}$ (using a modified version of the KOCA algorithm). The organizing process can be divided into four (04) phases, Initialization, the Construction of k-clustering, data dissemination and maintenance of the topology.

\section{E. Multihop-LEACH amlior}

J S Rauthan and S. Mishra [5], in 2012 ,proposed multihopLEACH improved protocol, which is one of the routing algorithms. The basic operation of multi hop-LEACH is similar toLEACHprotocol.There are two majorchanges in the multi hop-LEACH protocol compared to LEACH protocol. The multi-jump is applied in both the inter. and intra. Cluster communication.In this enhanced version of multi hop-LEACH protocol, the cluster contains; $\mathrm{CH}$ (responsible only to send the data that is received by the cluster members at the SB), vice$\mathrm{CH}$ (the node becomes acluster of $\mathrm{CH}$ incase $\mathrm{CH}$ dies),cluster nodes(collect data from the environment and send to $\mathrm{CH}$ ).

\section{F. PUCMR (Partition Based Unequally Clustered Multi-Hop Routing Protocol)}

U. Hari and Chris Johnson A[6], in 2013, state that the PUCMR protocol and unlike other uneven clusters based protocols, not only reduces the hot spot problem, but the issue of the unequal distribution of cluster-head is also eliminated. The proposed algorithm uses PUCMR energy, the degree of a node and the distance from center of gravity for the selection of cluster-Head and provides better position in the network.

Simulation results show that this approach extends the network lifetime.

\section{G. Assisted-LEACH}

Sunkara Vinodh Kumar and Ajit Pal,[7], in 2013, proposed the protocol Assisted-LEACH(A-LEACH) and declare that he has reached the level decreased and uniform distribution of the energy dissipated by the separation of routing tasks and aggregation of data.Heintroduced the concept of helper nodes(Nodes Helper) who assist Cluster Heads for Multi-hop routing.This algorithm has been, developed to facilitate energy efficiency,the configuration of the multi-hop route helper nodes to reach the base station.

\section{COMPARISON AND SYNTHESIS}

\section{A. Comprative Table}

The batch protocols that we have chosen, reports of recent work.Our comparison was, based on comparative factors shown in the table above. 
TABLE I: Terminology

\begin{tabular}{|l|l|}
\hline Symbole & Signification \\
\hline CL & Clustering use \\
\hline NC & Nature of generated clusters \\
\hline EC & Cluster Election \\
\hline RC & Cluster-Heads Rlection \\
\hline ER & $\begin{array}{l}\text { Consideration of node's Residualen ergy when } \\
\text { selecting CH }\end{array}$ \\
\hline CaC & Intra-Cluster Communication \\
\hline CrC & Inter-Cluster Communication \\
\hline ECH & Powers upporta large-scale network \\
\hline GT & Use of Gatewaynodes \\
\hline DA & Data Aggregation \\
\hline
\end{tabular}

\section{B. Synthesis}

In the previous sections, we have established a state of the ar ton many study protocols; we developed a comparative table based on a number of comparative indicators. To this end, we selected khLC Hand Assisted-LEACH protocols as the most power ful because the yrespond favorably to strong majority of the criteria on which we supported this study

\section{The Study Protocol}

\section{A. LEACH (Low Energy Adaptive Clustering Hierarchy)}

\section{Definition}

Heinzelman introduced a classification algorithm for sensor networks, called Low Energy Adaptive Hierarchical Clustering (Leach). LEACH is the first hierarchical cluster-based routing protocol for WSN. The advantage of this protocol is that it reduces the number of nodes that communicate directly with the base station and this by forming groups of clusterheads. Then the other neighboring nodes connect and become members of that cluster, and consume a minimum of energy.

\section{B. The operation mode of LEACH}

The protocol takes place in rounds that have approximately the same pre-determined time interval. Each cycle begins with an initialization phase followed by transmission phase. The duration of the communication phase is longer than that of the construction phase (initialization) to minimize the overhead.

1) The Initialization Phase: The purpose of this phase is the construction of clusters by choosing leaders $(\mathrm{CH})$ and setting the media to the access policy within each group

2) The Transmission Phase: This phase is longer than the previous one; it allows the collection of sensored data, using TDMA scheduler. The members transmit their data captured for their own slots; allowing them to turn off their communication interfaces outside their slots to save energy. This data is then aggregated by the $\mathrm{CH}$ that merges, compresses, and sends the final result to the base station.

After extinction of the $\mathrm{CHs}$, the network will move to a new round. This process is repeated until all network nodes will be selected $\mathrm{CH}$.

$$
\tau(n)= \begin{cases}\left.\frac{p}{1-p(r} \quad \bmod \frac{1}{p}\right) & \text { si } n \in G \\ 0 & \text { sinon }\end{cases}
$$

TABLE II: Comparison

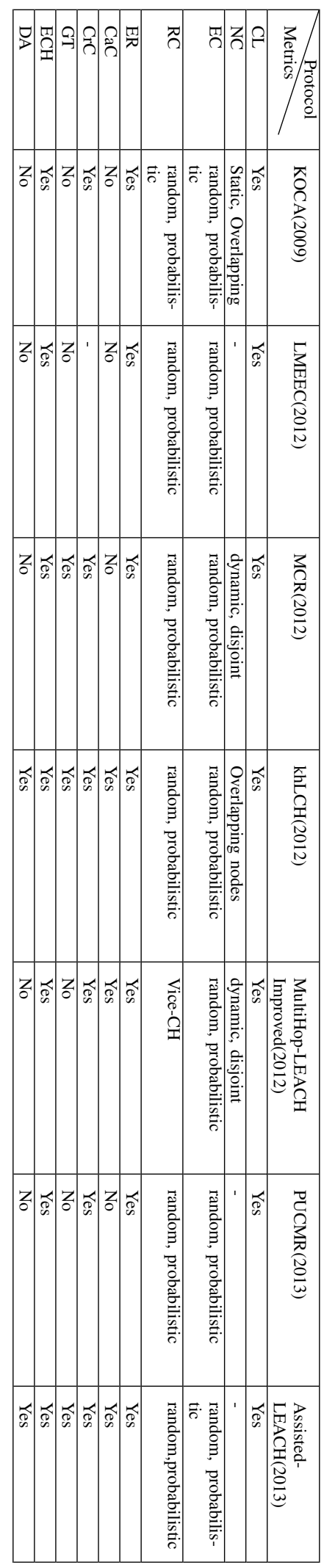

With: 
- $\mathrm{P}$ : is the desired percentage of $\mathrm{CH}$ that is to say selected as $p=0.05$ for optimal condition.

- $\quad r$ : current iteration.

- $\mathrm{G}$ : is the set of nodes that were not $\mathrm{CHs}$ at $(1 / p)$ previous

C. Assisted-LEACH (Low Energy Adaptive Clustering Hierarchy)

\section{Protocol Overview}

The Assisted-LEACH Protocol sometimes called ALEACH, includes the following sub-steps:o Selection of Cluster-Heads $(\mathrm{CH})$

- Creation of Clusters

- $\quad$ Selecting Helper Nodes

- The routing configuration

- Perception, aggregation and routing

\section{The operating mode}

In most clustering protocols, the entire load aggregation routing of data is done by the Cluster-Heads. The LEACH protocol transmits aggregated data directly by the cluster heads to the base station. This shortens the lifetime of the network. The concept of Helper Nodes was introduced, where a node closer to the base station in each cluster is assigned a routing task, while the cluster-Heads aggregate data. For the formulation of the route for helper nodes to reach the base station, each helper node is selected as the next hop; it is the nearest node to the base station among all neighboring helper nodes.

$$
T(n)= \begin{cases}\frac{p}{1-p\left(r \quad \bmod \frac{1}{p}\right)} & \text { si } n \in G \\ 0.5 \frac{\left.p \bmod \frac{1}{p}\right)}{1-p(r \quad} & \text { si } n \in H \\ 0 & \text { sinon }\end{cases}
$$

With:

- $\quad$ P: desired percentage of Cluster-Head

- $\quad \mathrm{r}$ : the current iteration in the operation protocol

- $\mathrm{G}$ : set of nodes that onttni-Cluster Head Nodes nor Helper in $[1 / p]$ past iterations.

- $\quad H$ : set of nudsqui not been ontpas Cluster-Heads but who played the role of helper nodes in $[1 / p]$ past iterations

\section{Simulation AND Results Interpetation}

\section{A. Presentation of NS2 Network Simulator}

Network Simulator is a discrete event simulator for networks and is mainly used for the simulation of all levels of communication protocols, providing support for wired as well as wireless networks. It was designed in $\mathrm{C}++$ and provides a simulation interface through otcl, an object-oriented language Tcl. The user must describe the network topology by writing otcl scripts that are then executed by the NS-2 main program
TABLE III: Simulation Parameters

\begin{tabular}{|l|l|}
\hline Parameters & Dimenssion \\
\hline Area & $100 \times 100$ \\
\hline Number of nodes & 10 \\
\hline Nodes initial energy & 2 joules \\
\hline Percentage of Cluster & 0.1 \\
\hline Energy consumed at data reception & $\mathrm{W}$ \\
\hline Energy consumed at data transmission & $\mathrm{W}$ \\
\hline Antenna Model & Omni Antenna \\
\hline Type of Traffic & CBR \\
\hline CBR Package Size & 32 octets \\
\hline Communication Model & Bi direction \\
\hline
\end{tabular}

\section{B. Experience and Discussion}

After completing our program ".tcl 'related to protocols and A-LEACH LEACH on NS2.35 we raised output the graphs below which and the respective interpretations are: He graph

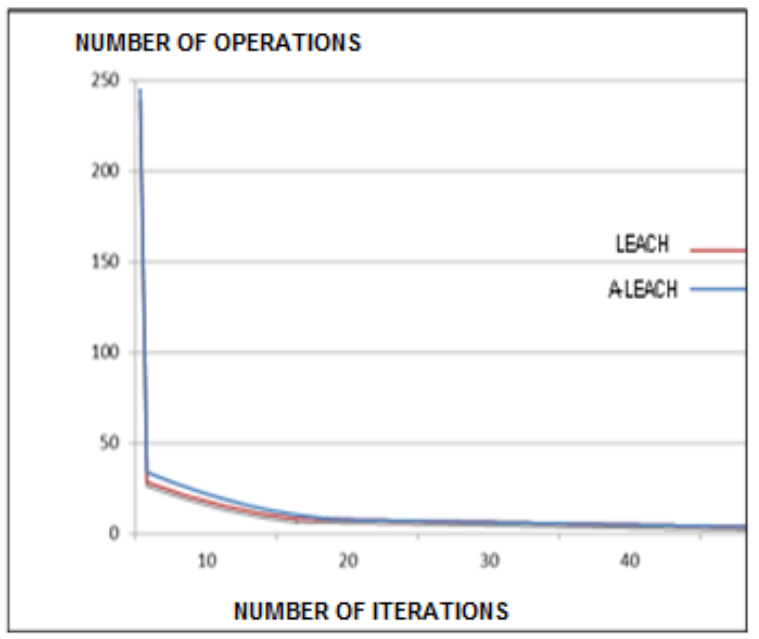

Fig. 2: Activity Task Manager

above represents the activity of the Task Manager of both LEACH and A-LEAH protocols

$\mathrm{x}$-axis; we have the number of iterations.

In ordinate, we have the number of operations performed by the scheduler.

Sending messages is done on a sliding scale, hence the reason why at the start of network deployment, the task

manager took charge of time allocation to serve all the nodes, but as network nodes disappear during the iterations, the scheduler increasingly lightens its operations until vacancy with the expiry of all nodes.

The results obtained from the trace file of the two routing protocols (LEACH and A-LEACH) are almost identical. They explain why the Task Managers of both protocols start with a total of 225 operations.

Then the LEACH manager begins to decrease gradually until it reaches 2 through the 37 th iteration. At the 38th 
iteration, it ends up with one and only one operation until the 51st iteration with network extinction, which means that at this stage it only manages itself.

The manager of A - LEACH begins to decrease gradually until it reaches 2 through the 39th iteration. And at the 40th iteration, it ends up with one and only one operation until the 53rd iteration with network extinction, which means that at this stage, as in $\mathrm{LEACH}$, it only manages itself. The graph

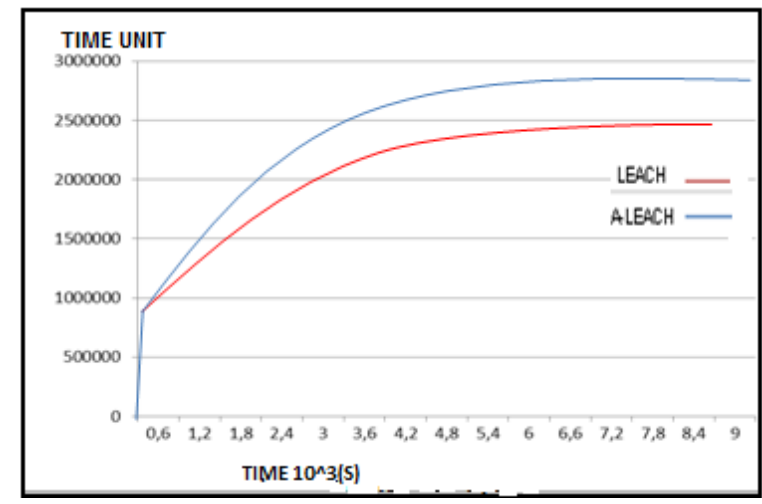

Fig. 3: the packages sent

above illustrates the concept of traffic communication between the cluster nodes and the cluster Head At X-axis, we have the time with 103 as a time unit.

In ordinate, we have the number of bits sent from the cluster and Head Node Helper. Sending messages is done progressively, hence the reason for the network stability in its early life and the disappearance of its nodes as a result of intensive messages ending. We collected figures from the trace file that shows for:

LEACH: 0 bits that start with the launch of the network, the number of packets sent from the cluster continues to rise, reaching 2.404 million bits sent after 780 time units, and remains stationary after explaining that there are more packet exchange due to the disappearance of all nodes.

A-LEACH: 0 bits that start with the launch of the network, the number of packets sent from the cluster continue to increase, to reach 2.668 million bits sent after 800 time units, and remains stationary until there is no more packet exchange due to the disappearance of all nodes.

The results obtained show that the packages sent by the cluster nodes using the A-leach protocol are growing compared to those sent by the nodes that use the LEACH protocol and this is because all nodes using protocol A-leach pass in a standby mode to reduce energy consumption.

The above graph illustrates the notion of life of nodes and that of the network. At $\mathrm{x}$-axis, we have time.

In ordinate we have the number of operational nodes.

Our graph and the results retrieved from the log file on the network life reflect the following results:

For Leach: results show network stability on energy consumption and preserving all of its nodes for almost $0.88 *$

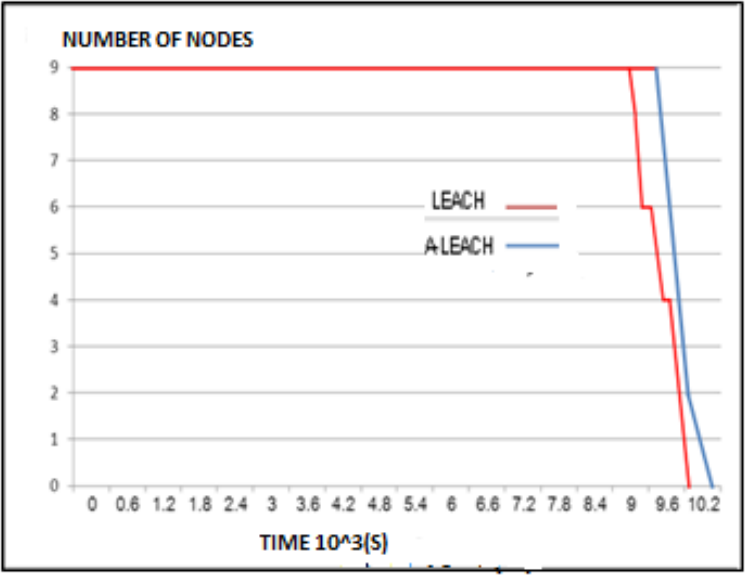

Fig. 4: lifetime

$103 \mathrm{~s}$ after which the network starts to record the gradual disappearance of its nodes to total extinction time in an approximate of $1.02 * 103 \mathrm{~s}$.

For A-leach: results shows network stability on energy consumption and preserving all of its nodes for almost 0.96 * $103 \mathrm{~s}$ after which the network starts to record the gradual disappearance of its nodes to total extinction in an the approximate time of $1.06 * 103 \mathrm{~s}$.

Consequently the life of a network using the protocol A-Leach is longer compared to a network using the Leach protocol.

\section{Synthesis}

Based on simulation results, we have shown that the ALEACH protocol improves energy dissipation within the clusters, increases energy gain, and extends the network lifetime compared to LEACH protocol.

So the A- LEACH protocol provided the best value because it increases network lifetime

\section{CONCLUSION}

Research in the field of sensor networks.

Is in full swing. Several routing protocols have been.

Developed in recent years. In this article we revised some routing protocols, with the aim of making a study of the performance of the latter.

We thought it useful to give an overview of the parameters (Metrics) used in the literature. We have extracted the better measures to measure performance in terms of Loss of packets and to decide the best of them under Conditions.

The work we have done (simulation under NS-2),

Allowed us to see the impact of many Nodes (or density), the energy consumed by the nodes and the variation of the scale, the rate of loss for protocols LEACH and A-LEACH 


\section{REFERENCES}

[1] S. Koteswararao, M. Sailaja et T. Madhu, "Implementation of Multihop Cluster based Routing Protocol for Wireless Sensor Networks", International Journal of Computer Applications (0975 - 8887)Volume 59- No.8, pp 2-5, 2012.

[2] Moustafa A. YOUSSEF, Adel YOUSSEF, Mohamed F. YOUNIS, "Overlapping MultihopClustering for Wireless Sensor Networks", IEEE Transactions On Parallel And DistributedSystems, Vol. 20, No. 12, 2009.

[3] ManelKhelifi, AssiaDjabelkhir, "LMEEC: Layered Multi-Hop Energy Efficient Cluster-basedRouting Protocol for Wireless Sensor Networks", ReSyD, Doctoral School in Computer Science UAMB, Bejaia university, Algeria IEEE Transactions On Parallel And DistributedSystems,pp $1-2,2012$.

[4] Khaled BOUCHAKOUR, "Routage hirarchique sur les rseaux de capteurs sans fil: Protocole KhLCH (K-hop LayeredClusteringHierarchy)", MEMOIRE Prsent pour l'obtention d'un diplme de MAGISTER EN INFORMATIQUE, pp 2-5, 2012.

[5] J. S. Rauthan, S. Mishra, "An improved Cluster Based Multi-hop Routing in Self-Organizing Wireless Sensor Networks", International Journal of Engineering Research Technology (IJERT) Vol.1, Issue 4, June - 2012.

[6] U. Hari, Chris Johnson A" A Partition BasedUnequallyClustered MultiHop Routing Protocol forWireless Sensor Networks", International Journal of Engineering ResearchTechnology (IJERT) Vol. 2 Issue 5, pp1714-1718, 2013

[7] SunkaraVinodh Kumar, Ajit Pal, " Assisted-Leach (A-Leach)Energy Efficient Routing Protocol for Wireless SensorNetworks", International Journal of Computer and Communication Engineering, Vol. 2, No. 4, pp 420-424,2013.

[8] Herman D. Hughes," Adaptive QoS Routing by Cross-Layer Cooperation in Ad Hoc Networks," EURASIP Journal on Wireless Communications and Networking", pp 661-671, 2005

[9] Draves, Richard and Padhye, Jitendra and Zill, Brian, "Routing in Multiradio, Multi-hop Wireless Mesh Networks", Proceedings of the 10th Annual International Conference on Mobile Computing and Networking, vol MobiCom ’04, pp 114-128, 2004. 